\title{
RESEARCH
}

Open Access

\section{Culture-enriched human gut microbiomes reveal core and accessory resistance genes}

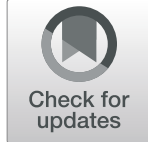

Frédéric Raymond ${ }^{1,2^{*}}$ (D), Maurice Boissinot ${ }^{3}$, Amin Ahmed Ouameur ${ }^{3}$, Maxime Déraspe ${ }^{3,5}$, Pier-Luc Plante ${ }^{3,5}$, Sewagnouin Rogia Kpanou ${ }^{3,5}$, Ève Bérubé ${ }^{3}$, Ann Huletsky ${ }^{3}$, Paul H. Roy ${ }^{3,7}$, Marc Ouellette ${ }^{3,4}$, Michel G. Bergeron ${ }^{3,4}$ and Jacques Corbei $i^{3,5,6}$

\begin{abstract}
Background: Low-abundance microorganisms of the gut microbiome are often referred to as a reservoir for antibiotic resistance genes. Unfortunately, these less-abundant bacteria can be overlooked by deep shotgun sequencing. In addition, it is a challenge to associate the presence of resistance genes with their risk of acquisition by pathogens. In this study, we used liquid culture enrichment of stools to assemble the genome of lowerabundance bacteria from fecal samples. We then investigated the gene content recovered from these cultureenriched and culture-independent metagenomes in relation with their taxonomic origin, specifically antibiotic resistance genes. We finally used a pangenome approach to associate resistance genes with the core or accessory genome of Enterobacteriaceae and inferred their propensity to horizontal gene transfer.

Results: Using culture-enrichment approaches with stools allowed assembly of 187 bacterial species with an assembly size greater than 1 million nucleotides. Of these, 67 were found only in culture-enriched conditions, and 22 only in culture-independent microbiomes. These assembled metagenomes allowed the evaluation of the gene content of specific subcommunities of the gut microbiome. We observed that differentially distributed metabolic enzymes were associated with specific culture conditions and, for the most part, with specific taxa. Gene content differences between microbiomes, for example, antibiotic resistance, were for the most part not associated with metabolic enzymes, but with other functions. We used a pangenome approach to determine if the resistance genes found in Enterobacteriaceae, specifically E. cloacae or E. coli, were part of the core genome or of the accessory genome of this species. In our healthy volunteer cohort, we found that E. cloacae contigs harbored resistance genes that were part of the core genome of the species, while E. coli had a large accessory resistome proximal to mobile elements.
\end{abstract}

Conclusion: Liquid culture of stools contributed to an improved functional and comparative genomics study of less-abundant gut bacteria, specifically those associated with antibiotic resistance. Defining whether a gene is part of the core genome of a species helped in interpreting the genomes recovered from culture-independent or culture-enriched microbiomes.

Keywords: Microbiome, Antibiotic resistance, Pangenome, Metagenomics assembly comparative genomics

\footnotetext{
* Correspondence: frederic.raymond@fsaa.ulaval.ca

'École de nutrition, Faculté des sciences de l'agriculture et de l'alimentation, Université Laval, Québec City, Canada

${ }^{2}$ Institut sur la nutrition et les aliments fonctionnels, Québec, Canada

Full list of author information is available at the end of the article
}

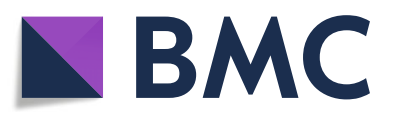

(c) The Author(s). 2019 Open Access This article is distributed under the terms of the Creative Commons Attribution 4.0 International License (http://creativecommons.org/licenses/by/4.0/), which permits unrestricted use, distribution, and reproduction in any medium, provided you give appropriate credit to the original author(s) and the source, provide a link to the Creative Commons license, and indicate if changes were made. The Creative Commons Public Domain Dedication waiver (http://creativecommons.org/publicdomain/zero/1.0/) applies to the data made available in this article, unless otherwise stated. 


\section{Background}

The widespread use of antibiotics has been accompanied by the appearance and dissemination of antibiotic-resistant bacteria, which is an alarming public health problem $[1,2]$. Moreover, antibiotics not only affect the pathogenic bacteria but also have profound effects on commensal bacteria involved in human health [3]. It has been proposed that the human gut microbiota serves as a reservoir of antibiotic-resistance genes (resistome) [4]. Genomic approaches substantially increased our knowledge about short- and long-term impact of antibiotics on the composition and functions of human gut microbiota [5-8]. Antibiotics can affect low-abundance taxa [9], thus requiring increased sequencing efforts for proper genome assembly and identification of strain and functions $[10,11]$.

While researchers routinely rely on culture-independent sequencing approaches to study the microbiome [12], there is a need to include cultivation techniques, combined with genome sequencing, to uncover less-abundant microorganisms, including bacteria, archaea, and fungi [13-15]. Culture on different media in Petri dishes, followed by colony picking, allowed the discovery and sequencing of the genome of hundreds of new species $[16,17]$. This culture-based approach can potentially allow the characterization of bacteria that are less abundant in the microbiota but still play a significant role in maintaining health [18]. Similarly, Lau and collaborators have grown microbiota on 33 solid media, both under aerobic and anaerobic conditions, to capture the diversity of the microbiome through culture [19]. Others have grown bacteria on solid or in liquid culture media to complement microbiome sequencing and to find culture conditions that allow the obtention of an accurate community representation of the complete microbiota $[20,21]$.

In this work, we set out to go further in the use of microbial culture as a complementary tool for microbiome analysis. We cultured stool samples in liquid media to complement information from cultureindependent microbiome (CIM) sequencing, thus allowing deep shotgun sequencing of culture-enriched microbiome (CEM). CEM permitted metagenome assembly of selected subcommunities from the gut microbiome. We chose the MCDA broth [22] as a base medium under two atmospheric conditions (aerobic with 5\% CO2 and anaerobic), and in the presence or absence of the antibiotic cefoxitin, a second-generation cephalosporin. We investigated how these different culture conditions allowed the metagenomic assembly of a greater segment of the gut microbiome. Deeper insight on the gut resistome was obtained from the CEM, especially when using a pangenome perspective to interpret the antibiotic resistance genes found in Enterobacteriaceae.

\section{Methods}

\section{Ethics statement and sampling}

The study protocol was approved by the ethical committee of the CHU de Québec-Université Laval. Informed written consent was obtained from participants. Healthy volunteer stool samples used in this study have been described in previous work [9].

\section{Study design}

The samples used in this study were collected from healthy participants that received oral doses of the antibiotic cefprozil for 7 days as described [9]. All samples were brought to the laboratory within $2 \mathrm{~h}$ of collection and placed immediately in an anaerobic chamber for processing. Samples were collected before antibiotic treatment (day 0), at the end of the treatment (day 7), and 3 months later (day 90). Culture-independent microbiome (CIM) sequencing was performed on these stool samples. Description of the collection, DNA extraction, and sequencing of CIM was described by Raymond and collaborators [9]. The same stool samples were used for culture-enriched microbiome (CEM) sequencing as described in the next section.

\section{Cultivation and DNA extraction}

Stool samples from 24 healthy volunteers, harvested at day 0 and day 7 were used for the generation of CEM. The fecal material (1 g) was suspended in $15 \mathrm{ml}$ PBS containing $0.1 \%$ cysteine (PBSc) and supernatant was obtained as previously described [23]. The supernatant was used to inoculate MCDA broth, which is based on brain-heart infusion (BHI) broth supplemented with haemin, vitamin K, L-cystine, lactate, and pyruvate [22]. Four CEM conditions for each sample were used in this study: (i) maximum enrichment broth anaerobic culture (MEB-ANA), (ii) maximum enrichment broth aerobic culture (air enriched with 5\% $\mathrm{CO}_{2}$ ) (MEB-CO2), (iii) anaerobic culture in the presence of $32 \mu \mathrm{g} / \mathrm{ml}$ cefoxitin (FOX-ANA), and (iv) aerobic (with $5 \% \mathrm{CO}_{2}$ ) culture in the presence of $32 \mu \mathrm{g} / \mathrm{ml}$ cefoxitin (FOX-CO2). Anaerobic cultures were incubated in a Bactron anaerobic chamber (Sheldon Manufacturing, Inc., Cornelius, Oregon, USA). Cultures were incubated at $35^{\circ} \mathrm{C}$ for 7 days to favor the growth of bacteria with longer generation time [23]. All cultures were conserved at $-80^{\circ} \mathrm{C}$ in the presence of $15 \%$ glycerol.

Crude DNA extracts were obtained from $1.5 \mathrm{ml}$ of frozen cultures using the BD GeneOhm ${ }^{\text {rM }}$ Lysis Kit as recommended by the manufacturer (BD Diagnostics-GeneOhm, Québec, Canada). Genomic DNA was purified using the Biosprint $^{\mathrm{TM}} 15$ DNA Blood Kit (Qiagen, Mississauga, Ontario, Canada) on a KingFisher ${ }^{\oplus}$ ML instrument according to the manufacturer's instructions (Thermo Fisher Scientific, Waltham, MA, USA). The quantity and quality of isolated DNA were measured using a NanoDrop ND-1000 
spectrophotometer (Thermo Fisher Scientific, Waltham, MA, USA), agarose gel electrophoresis, and a QuantiFluor ${ }^{\circ}$ dsDNA System (Promega). Extraction of CIM also included a bead-beating step and was performed with $\mathrm{MO}$ BIO PowerMax Soil DNA Extraction Kit (MO BIO Laboratories, Carlsbad, CA, USA) using a modified protocol [9].

\section{Shotgun sequencing}

For each sample, $50 \mathrm{ng}$ of purified genomic DNA were used to construct a sequencing library using a Nextera DNA sample preparation kit (Illumina, San Diego, CA, USA). Library validation was performed using Agilent Bioanalyzer 2100 high sensitivity DNA chips (Agilent Technologies, Santa Clara, CA, USA). Eight libraries with different indexes were pooled in equimolar concentration $(2 \mathrm{nM})$ prior to cluster generation in a cBot system (Illumina). Each pooled sample was sequenced in one lane using an Illumina HiSeq 1000 high-throughput sequencer with $2 \times 101$ bp paired-end sequencing according to the manufacturer's instructions. A total of 24 lanes were sequenced for 192 libraries. An average of $3.2 \mathrm{~Gb}$ was sequenced per CEM culture condition for each sample, for a total of $611 \mathrm{~Gb}$. Sequences of culture-enriched microbiomes (CEM) are available in European Nucleotide Archive PRJEB28237. Sequencing and assembly statistics of CEM are shown in Additional file 1: Table S1. We also compared CEM sequences to culture-independent microbiome (CIM) sequences obtained from the same participants in a previous study $[9,11]$. An average of $12.6 \mathrm{~Gb}$ were sequenced per CIM sample for a total of $907 \mathrm{~Gb}$. Sequences of CIM are available in European Nucleotide Archive PRJEB8094.

\section{Bioinformatics}

All genomic paired-end reads were assembled and profiled for taxonomy and resistance genes using the RAY Meta 2.2.1 assembler [24, 25] with the same protocol and reference database as in previous work [9]. Statistical analysis was done with R 3.0.2. Gene finding, resistance gene annotation, and contig identification were performed as described in supplementary materials of Raymond et al. [9]. In summary, putative genes were aligned to the MERGEM antibiotic resistance gene database and were annotated as resistance genes when similarity was more than $70 \%$ of the amino acid sequence.

Diversity indices were computed using the Vegan $R$ package. EC number annotation was performed by comparing putative proteins to the Brenda database using CD-hit-2D with a $95 \%$ identity threshold [26, 27]. Scripts for EC annotation are available on GitHub (https:// github.com/fredericraymond/GeneAnnotationTools). Comparison of gene content was performed for putative protein sequences at a threshold of $85 \%$ identity using CD-HIT V4.7 [26].
For pangenome analysis, genome collections for selected species were downloaded from NCBI. Genomes were annotated for resistance genes using the same protocol as for CIM and CEM, using the MERGEM database as reference [9]. Comparison of CIM and CEM are based on the presence/absence of specific antibiotic resistance genes.

\section{Results}

\section{Culture of fecal samples allows the sequencing of less- abundant bacteria}

Stool samples were grown for 7 days under four culture conditions using the same base media (MCDA broth) and were sequenced using shotgun metagenomics to generate CEM. Previously published culture-independent microbiome (CIM) sequencing from the same stool samples was used for comparison [9]. Taxonomic profiling shows that CEM sequencing gives a different portrait of communities compared to CIM (Fig. 1, Additional file 2: Figure S1 and Additional file 3: Table S2). We used the Shannon diversity index to compare the overall distribution of species observed in CIM and in CEM under the four culture conditions (Additional file 2: Figure S1A). Culture in the MEB-ANA and MEB- $\mathrm{CO}_{2}$ conditions had higher diversity than CIM, FOX-ANA, or FOX- $\mathrm{CO}_{2}$ conditions $(p=$ 3.7e-11, $t$ test). Culture in MEB conditions of samples from participants not exposed to antibiotics had significantly higher diversity than culture samples from participants exposed to cefprozil $(p=2.4 \mathrm{e}-6, t$ test $)$, which was also the case for CIM ( $p=0.004, t$ test). This difference was not observed when culture conditions included antibiotics ( $p=0.98, t$ test).

Of the 64 genera that were globally observed, 38 (59\% of all genera) were found at more than $1 \%$ of the community in culture only (Fig. 1). Indeed, the relative abundance of Escherichia is 100 to 1000 times higher in MEB- $\mathrm{CO}_{2}$ culture than in CIM. Enterococcus and Peptostreptococcus were detected in negligible amounts in CIM while they represented up to $1-10 \%$ in CEM. Difficult to culture Methanobrevibacter grew up to $5 \%$ in $\mathrm{CO}_{2}$ conditions. By contrast, Akkermansia, Alistipes, and Prevotella were 10-100 times more abundant in the CIM than under the CEM culture conditions. Interestingly, several genera observed in culture (Fig. 1, in bold), especially under the MEB- $\mathrm{CO}_{2}$ condition, were previously found to be associated with the mucus layer of the intestine [28].

Assembled metagenomes were annotated based on their k-mer similarity to known genomes thus enabling identification of their taxonomic provenance. The total assembly size of each species was calculated for all samples and conditions (Additional file 4: Table S3 and Additional file 5: Figure S2). Overall, 187 species had an assembly size greater than 1 Mbp for at least one condition and 22 species reached it 


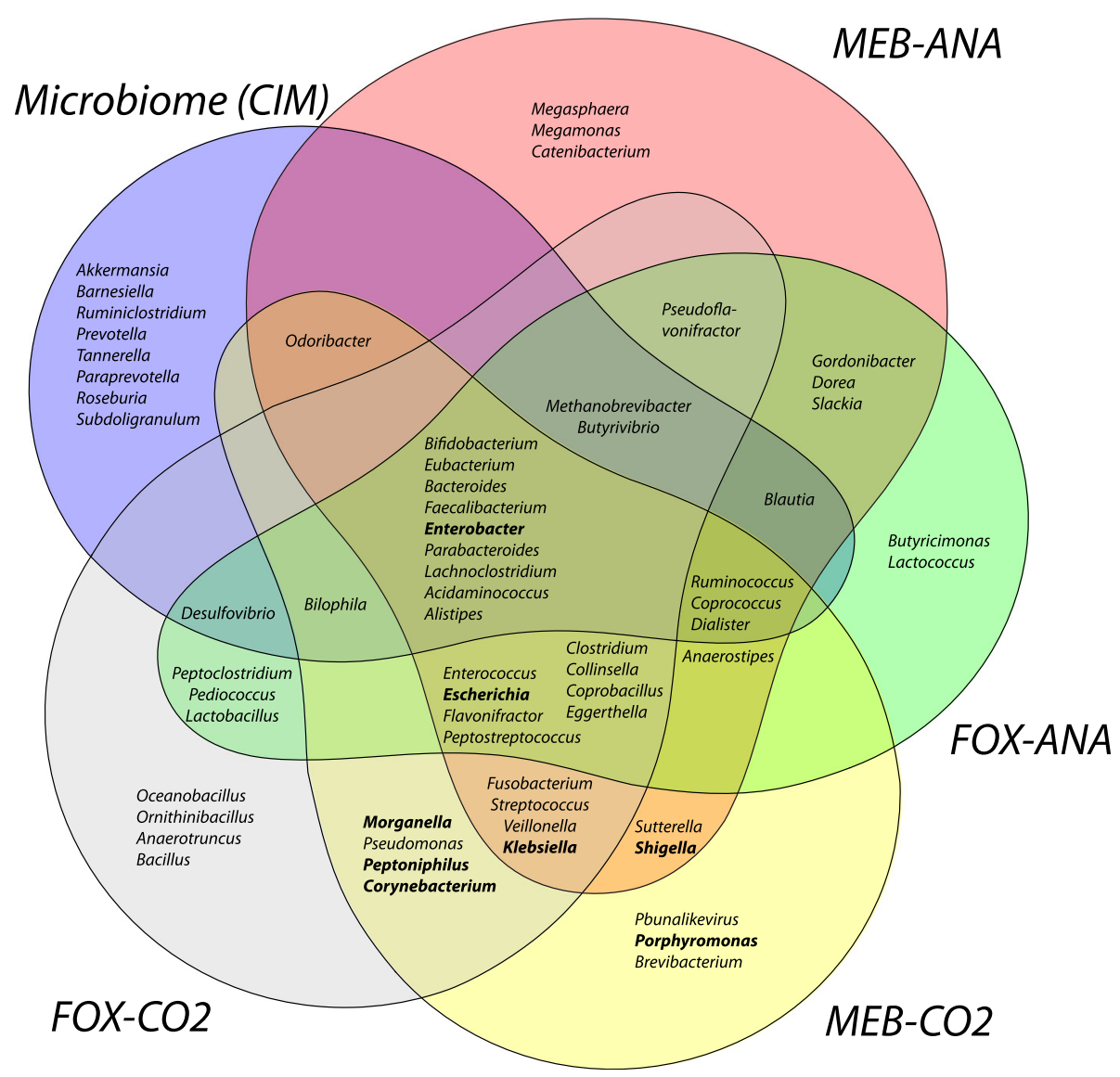

Fig. 1 Comparison of microbial genera observed in the four culture-enriched conditions and in the culture-independent microbiome metagenomes. Genera shown were at least 1\% of the microbial community in at least one sample of the five conditions that were sequenced. Genera in bold were identified as mucosally associated by Albenberg and collaborators [28]

only in CIM and 67 only in CEM. A total of 148 species had an assembly size greater than 2 Mbps (22 only in CIM and 48 only in CEM), 101 greater than 3 Mbps (29 only in CIM and 33 only in CEM), and 58 greater than 4 Mbps (15 only in CIM and 17 only in CEM). In all cases, more species were assembled above these thresholds in CEM than in CIM. Using culture approaches with stools allowed to sequence the genome of a greater number of species not sequenced originally. We could then perform a better evaluation of the gene content of taxa that are less abundant in the microbiome but grow well in selected culture conditions. The most notable example is the Enterobacteriaceae like Escherichia, which are well known antibiotic resistance gene carriers and likely have a significant impact on the global gut resistome.

\section{Gene content of culture-enriched microbiota}

We investigated how culture conditions affected the metabolic pathways observed in the CEM compared to CIM. Assembled CIM and CEM were thus annotated with genes associated with metabolic pathways, based on Enzyme Commission (EC) annotation (Fig. 2). We detected
1856 different enzymes (different EC numbers) in CIM samples while 2176 different enzymes were annotated in the CEM samples. Overall, 1828 enzymes were found both in CIM and in CEM. Although the sequencing depth and assembly size of CEM were respectively 4.2 and 2.5 times lower than CIM, 348 enzymes were unique to CEM and only 28 were specific to CIM. These enzymes belonged to a total of 170 metabolic pathways, 151 of which were found under all culture conditions and in CIM, in at least one sample (Additional file 6: Figure S3). Pathways not observed in all conditions were associated with the biosynthesis of secondary metabolites.

Enzymes were clustered based on their distribution in the CIM and CEM using k-means clustering. Each enzyme was also annotated with the taxonomic origin of the contigs on which it was found (Fig. 3a). The putative taxonomic origin of enzymes was coherent with clustering enzymes into 12 groups, and this clustering reflected the selection of taxa by culture (Fig. 3a, b). The proportion of samples positive for enzymes per conditions and clusters is shown in Additional file 7: Figure S4. In addition, metabolic pathway coverage was calculated for 


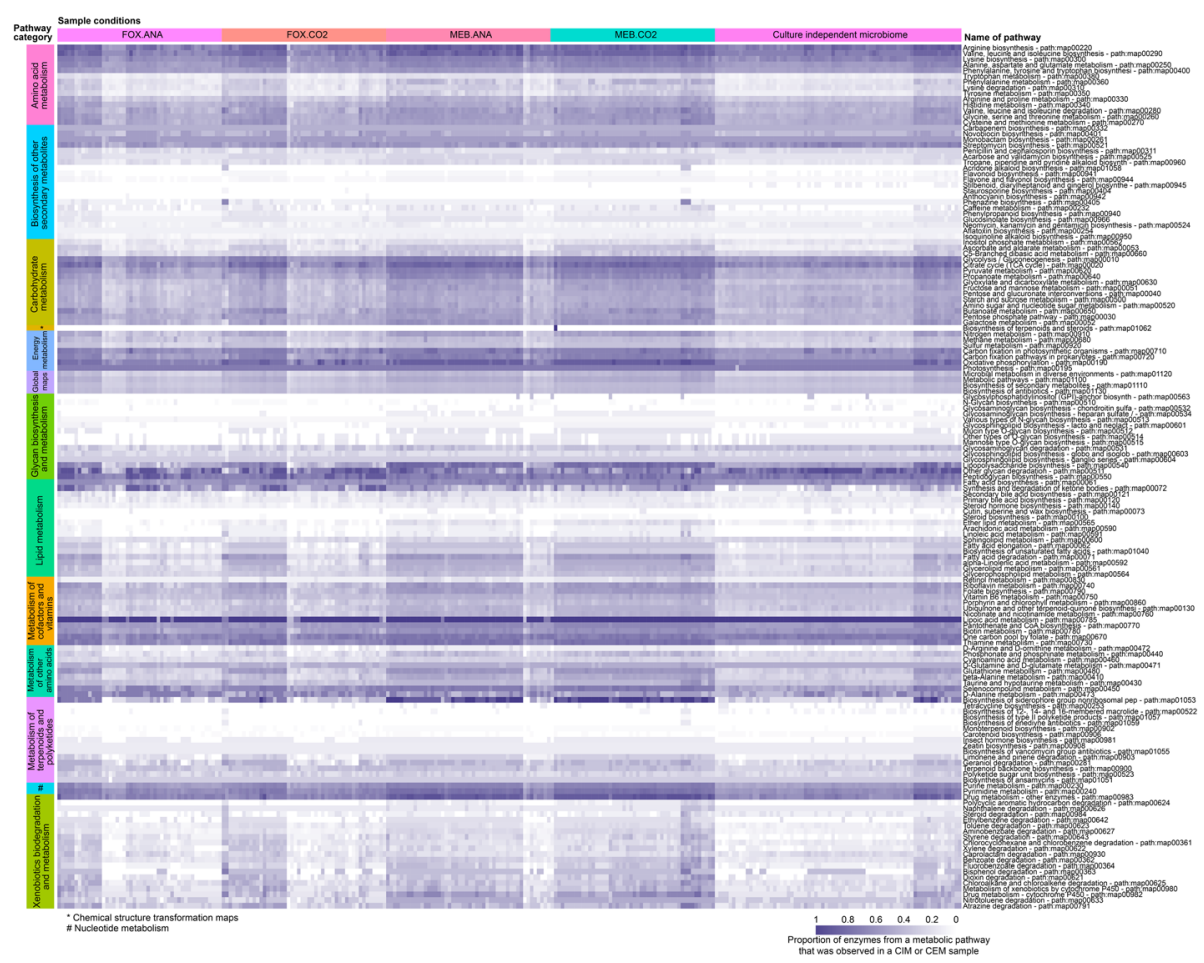

Fig. 2 Coverage of metabolic pathways in culture-independent and culture-enriched metagenomes. Coverage is the proportion of enzymes from a metabolic pathway that was observed in a sample. Metabolic pathways are hierarchically clustered within pathway categories, which are indicated by a color code. Columns of the heatmap are samples hierarchically clustered within CIM and CEM conditions

the 12 clusters (Fig. 3c). The most ubiquitous enzymes belonged to cluster 1 , from which 587 enzymes were shared by more than $81.4 \%$ of samples, both in CIM and CEM. These enzymes could be associated with approximately 230 genera. Pathway categories with lesser representation in cluster 1 were biosynthesis of secondary metabolites, glycan biosynthesis and metabolism, and metabolism of terpenoids and polyketides. Pathways absent from cluster 1 were most of the time represented in other clusters, suggesting that they would be taxa-specific. Although they were also observed in some CEM samples, enzymes from clusters 2 and 4 were strongly represented in CIM. The most frequent taxonomic origin of enzymes found in cluster 2 was Bacteroides while enzymes from Cluster 4 were observed in both Bacteroides and Enterobacteriaceae. Cluster 9 was associated with Enterococcus, which was mostly observed in CEM in the presence of cefoxitin. Interestingly, in cluster 10, three cultures under $\mathrm{CO}_{2}$-enriched atmosphere from the same participant, in the presence or absence of cefoxitin, yielded almost pure cultures of Pseudomonas aeruginosa. Cluster number 12 was not associated with any specific condition or taxa and included genes that were either rare in CIM or present in less-abundant bacterial species, which are difficult toassemble. Overall, these functional analyses suggest that metabolic pathways are strongly correlated with the taxonomic composition of microbial communities, a hypothesis supported by other studies $[29,30]$.

Nonetheless, the 1,581,710 putative genes stringently annotated as metabolic enzymes constitute only $7.3 \%$ of total genes detected in CIM and CEM. We thus generated clusters of orthologous genes (COG) using all $21,715,728$ predicted genes. We used a clustering threshold of 85\%, creating 2,009,496 COG. This approach allowed a comparison of the total gene content of microbial populations without function selection bias. The distribution of these COG between culture conditions and time points indicated a different distribution than that based on enzymes and pathways (Fig. 4). Indeed, while pathways and EC-annotated enzymes are in general present under all conditions, $48.9 \%$ of COG were specific to uncultured microbiomes and only $2.9 \%$ were observed in all four selective culture conditions and in the direct microbiomes. Also, $16.7 \%$ of COG were observed in a single CEM condition and time point. These results indicate that differences in microbiome gene content are for the most part not associated with metabolism-related genes, but with genes that have other functions. It explains in part why software that extrapolates metabolic pathways from $16 \mathrm{~S}$ metabarcoding data perform appropriately for metabolism, but cannot assess the accessory genome and thus do not explain all phenotypes. Indeed, 

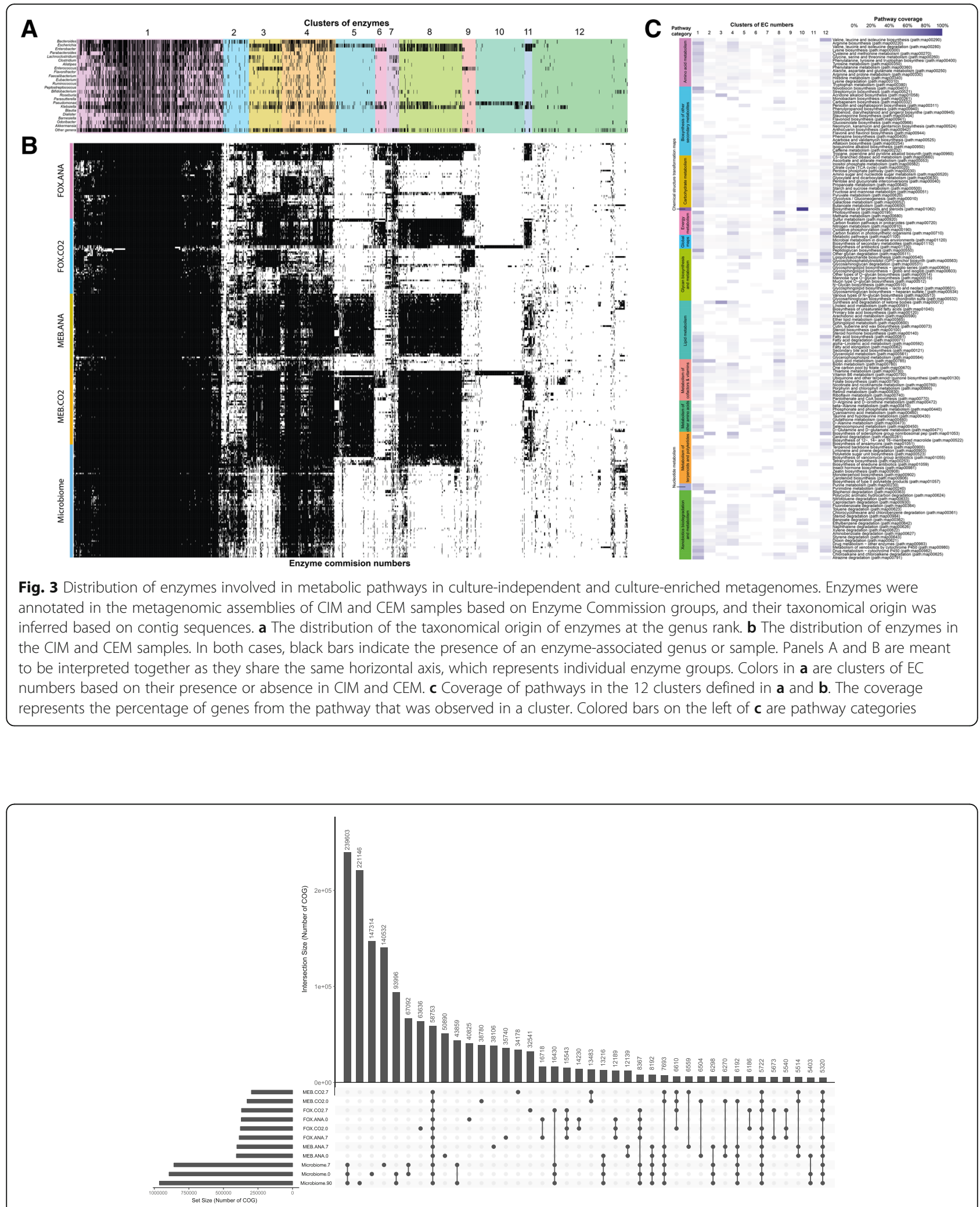

Fig. 4 Distribution of $85 \%$ identity clusters of orthologous genes (COG) in culture-independent and culture-enriched microbiomes. Presence of COG in a given intersect is shown as a black dot in the intersection table at the bottom of the figure. The barplot at the top represents the number of COG in each intersection. The barplot on the left indicates the number of COG in each sample condition. The number at the end of conditions identifies the timepoint of sample collection $(0,7$, or 90 days) 
the health impact of gut microbes could be mediated by accessory genes such as those conferring antibiotic resistance.

\section{Mining assemblies for antibiotic resistance genes}

We annotated a total of 13,218 putative resistance genes with at least $70 \%$ amino acid identity to genes known to confer resistance to antibiotics (Additional file 8: Table S4). These genes could be classified into 224 types. More than $98 \%$ of putative resistance genes were located on contigs for which taxonomic origin could be inferred (Additional file 9: Table S6 and Additional file 10: Table S7). A total of 3043 resistance genes were found near insertion sequences (Additional file 11: Table S5) and were assigned to 149 different types. Culture-enriched microbiomes allowed observation of 1.3 times more resistance genes than CIM.

Species with the highest number of resistance genes annotated when summing the assemblies of all tested conditions were Escherichia coli (5386 potential resistance genes on E. coli contigs), Enterobacter cloacae and related strain ST3 (2098), Staphylococcus aureus (297), Bacteroides uniformis (251), Campylobacter jejuni (229), Klebsiella pneumoniae (222), and Enterococcus faecalis (214). To evaluate the risk of these species harboring acquired antibiotic resistance genes in our antibiotic-exposed participants, we compared the antibiotic genes annotated on these contigs with the antibiotic resistance genes found in the pangenome of these species from publicly available datasets in NCBI Genomes (Table 1). Antibiotic resistance genes shared by more than $95 \%$ of the genomes of a species (the "soft-core" genes) were thus expected to be present in microbial communities that contain this species. Genes that are only found in a small subset of genomes, the flexible or accessory genome (5\% to $95 \%)$ and the rare genes $(<5 \%)$, are more likely the product of HGT and were considered potentially acquired accessory genes in the following analysis [31]. However, it is not possible to use this approach to determine if this acquisition is recent or ancient.

A total of 44 resistance gene types were found in contigs suspected of originating from E. cloacae based on k-mer content similarity. We investigated these genes using the pangenome of 432 publicly available sequences of $E$. cloacae genomes by annotating these genomes using the MERGEM database (Fig. 5, upper part). On average, E. cloacae genomes harbored 40 resistance genes. Thirty-four $E$. cloacae putative resistance genes found in CIM or CEM belonged to the core genome of this species, because they were found in more than $95 \%$ of the sequenced E. cloacae genomes (Fig. 5, lower part). Moreover, six of the non-core resistance genes found in our samples were the chromosomally encoded blaACT beta-lactamase genes (also annotated as blaMIR), which represent different alleles of a gene that is present in all strains. These alleles are linked to whole genome phylogeny of E. cloacae (Additional file 12:
Figure S5). The 3 remaining accessory resistance genes ( $m d t L$, fos $A, o m p F)$ were prevalent in the pangenome (present in more $70 \%$ of samples). Therefore, we propose that the E. cloacae strains observed in the participants after cefprozil exposure did not carry clinically important transferable resistance genes. Still, core resistance genes could have been involved in the thriving of E. cloacae in the microbiomes after antibiotic treatment [9]. It is of note that a large proportion of the reference $E$. cloacae genomes were from clinical isolates that were obtained through screening for multidrug-resistant Enterobacteriaceae [32]. This could explain why the reference $E$. cloacae genomes contained more resistance genes than the strains observed in the microbiome of healthy individuals.

We performed a similar analysis on contigs presumably originating from $E$. coli and found 68 families of resistance genes in putative $E$. coli contigs. On average, $E$. coli genomes harbored 46 resistance genes. We compared the putative resistance gene content of these contigs with the resistance genes found in 3346 E.coli genomes (Fig. 6). We also observed a large intrinsic resistome in E.coli, as 35 genes are shared by more than $95 \%$ of genomes. Two groups of blaAmpC genes were observed in the microbiomes and samples could be delineated based on alleles, which correlated with the population structure, a behavior similar to blaACT in E. cloacae (Additional file 13: Figure S6). However, by contrast to $E$. cloacae, 33 types of putative resistance genes were found in only a subset of microbiomes and were present in a limited number of the $E$. coli genomes $(<95 \%)$. Moreover, accessory resistance genes and mobile elements are physically closer on chromosomes in $E$. coli than in $E$. cloacae (Additional file 14: Figure S7). These results suggest that those genes are part of the accessory $E$. coli genome and could have been acquired by horizontal gene transfer.

A total of 197 putative resistance genes were potentially located in Enterococcus faecalis and belonged to 7 resistance gene types. We thus investigated how the resistance genes observed in CIM or CEM were related to those observed in E. faecalis (246) and E. faecium (119) genomes available in public databases. No annotated resistance genes were part of the core genome for these two species. Respectively, $73 \%$ of $E$. faecalis and $88 \%$ of $E$. faecium resistance genes were found in less than $5 \%$ of sequenced genomes, suggesting that they could have been acquired by horizontal gene transfer. Five putative resistance genes found in the microbiome were part of the accessory genome of E. faecalis, and two were rare genes in this population. Similarly, in E. faecium, 5 of the 7 observed resistance genes were part of the accessory genome, 1 was rare, and 1 (aac(6')-Ii) was not observed in $E$. faecium sequenced genomes.

We observed only accessory $(<95 \%)$ or rare $(<5 \%)$ resistance genes in Staphylococcus aureus genomic context 
Table 1 Comparison of antibiotics resistance genes from culture-independent and culture-enriched microbiomes to the pangenome of selected species

\begin{tabular}{|c|c|c|c|c|c|c|c|c|}
\hline & & $\begin{array}{l}\text { Bacteroides } \\
\text { uniformis }\end{array}$ & $\begin{array}{l}\text { Campylobacter } \\
\text { jejuni }\end{array}$ & $\begin{array}{l}\text { Enterobacter } \\
\text { cloacae }\end{array}$ & $\begin{array}{l}\text { Enterococcus } \\
\text { faecalis }\end{array}$ & $\begin{array}{l}\text { Enterococcus } \\
\text { faecium }\end{array}$ & $\begin{array}{l}\text { Escherichia } \\
\text { coli }\end{array}$ & $\begin{array}{l}\text { Staphylococcus } \\
\text { aureus }\end{array}$ \\
\hline \multirow[t]{5}{*}{$\begin{array}{l}\text { Reference } \\
\text { genomes }\end{array}$} & $\begin{array}{l}\text { Number of genomes of species for } \\
\text { panresistome construction }\end{array}$ & 14 & 458 & 432 & 246 & 119 & 3346 & 6632 \\
\hline & $\begin{array}{l}\text { Types of resistance genes found in } \\
\text { species (total resistome) }\end{array}$ & 29 & 29 & 225 & 79 & 172 & 296 & 112 \\
\hline & $\begin{array}{l}\text { Types of resistance genes present } \\
\text { in }>95 \% \text { genomes of species (core } \\
\text { resistome) }\end{array}$ & 0 & 2 & 34 & 0 & 0 & 35 & 12 \\
\hline & $\begin{array}{l}\text { Types of resistance genes present } \\
\text { in }<95 \% \text { and }>5 \% \text { genomes of } \\
\text { species (accessory resistome) }\end{array}$ & 9 & 5 & 35 & 21 & 21 & 23 & 18 \\
\hline & $\begin{array}{l}\text { Types of resistance genes present } \\
\text { in }<5 \% \text { genomes of species (Rare } \\
\text { resistome) }\end{array}$ & 20 & 22 & 156 & 58 & 151 & 238 & 82 \\
\hline \multirow[t]{6}{*}{$\begin{array}{l}\text { CIM and } \\
\text { CEM }\end{array}$} & $\begin{array}{l}\text { Number of CIM or CEM positive } \\
\text { for resistance genes found in the } \\
\text { context of species }\end{array}$ & 157 & 174 & 39 & 124 & 30 & 146 & 101 \\
\hline & $\begin{array}{l}\text { Types of resistance genes found in } \\
\text { the context of the species in CIM } \\
\text { or CEM (total resistome) }\end{array}$ & 9 & 3 & 44 & 7 & 7 & 68 & 10 \\
\hline & $\begin{array}{l}\text { Types of resistance genes found in } \\
\text { the context of the species in CIM or } \\
\text { CEM and present in }>95 \% \text { genomes } \\
\text { of species (core resistome) }\end{array}$ & 0 & 0 & 35 & 0 & 0 & 35 & 0 \\
\hline & $\begin{array}{l}\text { Types of resistance genes found in } \\
\text { the context of the species in CIM or } \\
\text { CEM and present in }<95 \% \text { and }>5 \% \\
\text { genomes of species (accessory } \\
\text { resistome) }\end{array}$ & 6 & 1 & 6 & 5 & 5 & 22 & 4 \\
\hline & $\begin{array}{l}\text { Types of resistance genes found in } \\
\text { the context of the species in CIM or } \\
\text { CEM and present in }<5 \% \text { genomes } \\
\text { of species (rare resistome) }\end{array}$ & 0 & 0 & 3 & 2 & 1 & 10 & 4 \\
\hline & $\begin{array}{l}\text { Types of resistance genes found in } \\
\text { the context of the species in CIM } \\
\text { or CEM and not found in } \\
\text { reference genomes }\end{array}$ & 3 & 2 & 0 & 0 & 1 & 1 & 2 \\
\hline
\end{tabular}

in the CEM and CIM and no resistance genes were part of the core $S$. aureus resistome. Further investigation of putative $S$. aureus contigs using blastn on NCBI indicate potential similarity with taxa such as uncultured bacteria, Enterobacter, Campylobacter, or Ruminococcus, or sequences from plasmids. This is concordant with the low relative abundance of Staphylococcus (from a background signal to $0.3 \%$ ) in the samples and suggests that the contigs identified as $S$. aureus could be wrongly annotated due to an incomplete reference database.

\section{Discussion}

This study was designed to investigate how different, but complementary, stool culture conditions allowed the sequencing and analysis of bacteria that could not be properly sequenced using deep culture-independent microbiome sequencing. Using the MCDA broth as culture media, we have incubated stool samples in anaerobic and $5 \% \mathrm{CO}_{2}$ conditions to determine how aerobic or anaerobic culture-enriched different bacteria. The two atmospheric conditions were also incubated in the presence or absence of the cefoxitin antibiotic to enrich for antibiotic-resistant bacteria. Overall, we found that sequencing broth culture of stool samples (CEM) allowed assembly of genomes that could not have been assembled using only culture-independent microbiome sequencing (CIM). For example, our CEM approach allowed assembly of the genome of Enterobacteriaceae, such as E. coli, E. cloacae, and K. pneumoniae, presenting abundances often lower than $1 \%$ in gut CIM. Indeed, E. coli had lower than $0.1 \%$ relative abundance in CIM, but was on average $15.2 \%$ of the community in MEB-CO $\mathrm{CO}_{2}$ and $6.2 \%$ in MEB-ANA. This was to be expected as E. coli, although not dominant in the gut microbiome, is well adapted for 


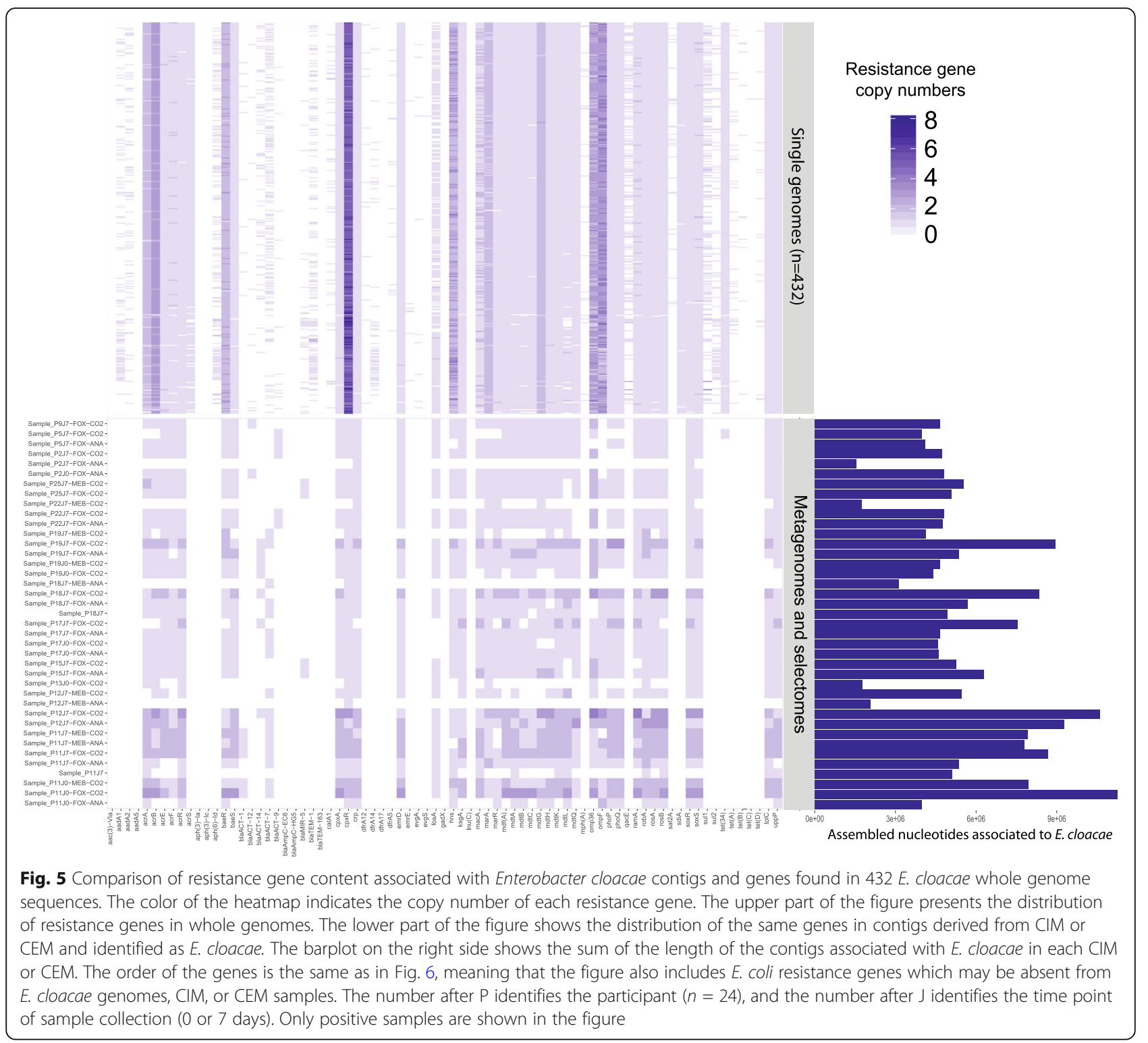

growth in vitro. The $5 \% \mathrm{CO}_{2}$-enriched atmosphere allowed the growth of aerobes such as Pseudomonas and Brevibacterium. Moreover, there is a core of genomes that can be observed in CIM and CEM.

Notable gut microbiome bacteria like Akkermansia or Prevotella were not observed in CEM while they were prevalent in CIM. We suspect that the culture conditions used in this study were not appropriate for these species, as they are known to be difficult to culture. In addition, sample collection, storage, and preparation may have affected the taxa that were cultured in CEM and account for some differences between CIM and CEM. The extraction method for CIM and CEM was different as extraction from feces and bacterial culture have different requirements. However, bead-beating was used for cell lysis in both cases. Exposure to oxygen may have affected obligate anaerobic bacteria during sample collection and culture preparation. The genome of these bacteria could then have been present in the fecal samples, but the microorganism may not have been able to grow in culture [33]. The composition of culture media would also affect the community composition. For example, MCDA broth did not include short-chain fatty acids, which are required for the grown of some anaerobic bacteria like Roseburia [33]. In addition, some bacterial species may have faster growth rates in the selected culture conditions, which may favor them in the obtained CEM compared to bacteria with slower growth.

Nonetheless, broth culture under $5 \% \mathrm{CO}_{2}$-enriched atmosphere, and anaerobic culture shared $57 \%$ of the genera found at more than $1 \%$ of the cultured community, many of these taxa being obligate anaerobes such as 


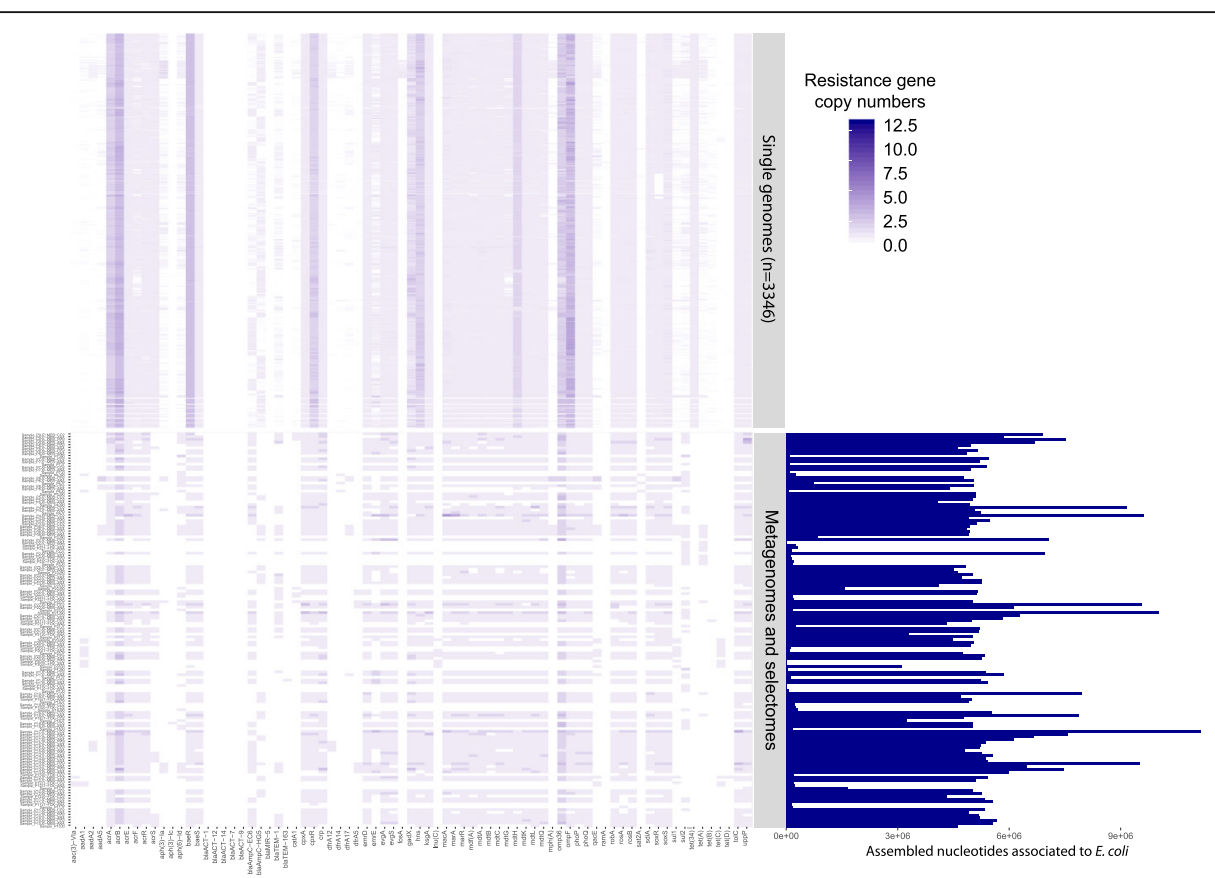

Fig. 6 Comparison of resistance gene content associated with Escherichia coli contigs and genes found in 3346 E. coli whole genome sequences. The color of the heatmap indicates the copy number of each resistance gene. The upper part of the figure presents the distribution of resistance genes in whole genomes. The lower part of the figure shows the distribution of the same genes in contigs derived from CIM or CEM and identified as E. coli. The barplot on the right side shows the sum of the length of the contigs associated with E. coli in each CIM or CEM. The order of the genes is the same as in Fig. 5, meaning that the figure also includes E. cloacae resistance genes which may be absent from $E$. coli genomes, CIM, or CEM samples. The number after $\mathrm{P}$ identifies the participant $(n=24)$ and the number after $\mathrm{J}$ identifies the time point of sample collection (0, 7, or 90 days). Only positive samples are shown in the figure

Parabacteroides, Ruminococcus, Porphyromonas, or Bilophila [28]. Although the medium does not contain oxygen reducing agents including those found in thioglycollate broth [34], oxygen reduction could be occurring. Indeed, through the growth of certain taxa in the community, oxygen at the bottom of the tube could be consumed or detoxified, thus allowing the growth of anaerobic bacteria. Since the 15-ml broth culture tubes were not shaken (nor stirred) and incubated for a period of 7 days, a diminishing $\mathrm{O}_{2}$ gradient might have been established, therefore explaining the apparent growth of anaerobes in $\mathrm{CO}_{2}$. In addition, bacteria thought to be strictly anaerobic could adapt to microaerobic conditions by modulating gene expression, as shown for Porphyromonas gingivalis [35].

The broth culture conditions used in this study permitted the growth of microbial communities that could include previously difficult to sequence taxa due to their low relative abundance in the microbiome. For example, the MEB- $\mathrm{CO}_{2}$ condition allows assembling the genomes of several genera that have been associated with the mucus layer of the intestine [28] or bacteria known to reside in the small intestine or in the interfold regions [36]. We thus suggest that the selection of culture media based on bacterial taxa of interest could allow for a deeper insight into specific subcommunities of the gut microbiome.
Moreover, the sequencing depth of CEM (3.2 Gb/CEM) was on average fourfold lower than CIM (12.6 Gb/CIM), thus four times less expensive than CIM to recover additional genomes. The use of carefully chosen selective media could prove a viable method for the metagenomic characterization of specific portions of the microbiota without invasive procedures and with reduced sequencing effort. This approach is complementary to culture-independent microbiome sequencing and can be performed after initial metagenomic profiling. In addition to recovering the genome of lower abundance bacteria, recovery of specific taxa in CEM would suggest that they were viable in the stool sample, a notion that cannot be inferred from CIM [37]. A major limitation of this growth-based approach is that culture limits our capacity to do a quantitative comparison of the relative abundance of bacteria between CEM. However, CIM or 16S metataxonomics would still retain this information and could be used as a reference.

Culture-enriched microbiome analysis allowed the evaluation of the gene content of specific subcommunities of the gut microbiome, as the assembly of CIM and CEM permitted the characterization of the functional profile of bacteria. We annotated metabolic pathway enzymes on the assembled contigs to determine which pathways were detected in CIM or CEM. Then, 
we identified the putative taxonomic origin of contigs to infer to which bacterial functions they were most probably associated. Contigs can be properly identified based on adequate representation of the gut microbiome in the reference genome database used for taxonomic profiling [25]. It remains possible that this identification could be biased by database composition or by horizontal gene transfer. We first observed that the four culture conditions and CIM sequencing shared a large pool of similar enzymes, which are associated with diverse microbial origins and functions. Some enzymes were associated with specific culture conditions and, for the most part, with specific taxa. This is in accordance with findings that show an association between the functions found in the microbiome and taxonomical distribution [29, 30]. However, other work indicates that mobile genes could often be structured according to ecological context rather than by taxonomy [38, 39], which is coherent with the overall distribution of genes, regardless of functions, between samples and conditions.

This last paradox is especially true for antibiotic resistance genes, which can both be intrinsic to the core genome of a species or acquired through horizontal gene transfer [40]. Studies of antibiotic resistance genes in the microbiome often consist in catalogs of genes, thus overlooking the difference in risk associated with genes prone to HGT compared to genes intrinsic to the core genome [41]. For example, in our previous work, we observed several antibiotic resistance genes associated with $E$. cloacae genomes reconstituted from metagenomic sequencing after exposure of volunteers to the antibiotic cefprozil [9]. However, quantifying the HGT potential of these genes was problematic, as short-read sequencing does not provide an easy way to determine whether genes are intrinsic or have been acquired by horizontal transfer. As the CEM approach allows assembly of a large array of genomes from Enterobacteriaceae, we developed a method to infer if antibiotic resistance genes were intrinsic or the result of potential HGT. First, we generated the panresistome of a species by annotating a collection of genomes obtained from public databases. Then, we compared the gene content of contigs identified as originating from this species with the panresistome of the species. This approach allowed identification of genes belonging to the core genome of a species, thus suggesting that they have a low probability of being mobile. After the analysis of the E. cloacae panresistome, we found that resistance genes from this species were associated with its core genome and not with potentially mobile elements. By contrast, the E. coli-related contigs contained numerous resistance genes belonging to the accessory genome, some of which were rare in the $E$. coli species population, suggesting that some E. coli resistance genes in the microbiome of our study participants could be mobile, or at least strain-specific. Genes belonging to the core genome of a species are often associated with essential functions. As antibiotics target core functions of the bacteria, many resistance genes can be part of the core genome of some species. For example, the blaACT of E. cloacae or blaAmpC of E. coli are part of the peptidoglycan biosynthesis machinery in Enterobacteriaceae, thus conferring upon these bacteria intrinsic resistance to some antibiotics [42]. In contrast, accessory resistance genes are optional and not essential for the survival of a species in normal conditions. These can however be selected in the presence of a selective agent such as an antibiotic.

The pangenome method can also diagnose the assembly of a species, as the coherence between the core genes found in reference genomes and those found in metagenomes indicate that this species has an appropriate sequencing coverage. As seen with $S$. aureus, discordances could suggest either an incorrect annotation of contigs or an inability to assemble the complete genome of a species. Still, the CEM method has some drawbacks such as inability to take into account resistance genes in contigs that were not associated with a given species because of low coverage, absence of representative strains in reference databases or presence of genes in contigs associated with other species because of HGT. Moreover, the origin of the reference genomes should be taken into account during the interpretation of pangenome-derived analysis. Clinical isolates from a species can harbor a different resistome than commensals. For instance, a large proportion of the Enterobacter cloacae genomes used as a reference in this work came from a study screening for multidrug-resistant Enterobacteriaceae [32]. As such, this E. cloacae genome collection may not be fully representative of the diversity of this species. In contrast, the microbiomes included in this study are from healthy individuals without underlying conditions; therefore, the E. cloacae strains observed in CIM and CEM may not be pathogenic. Still, this approach allowed to determine if changes in antibiotic resistance genes in microbiomes were linked to taxonomy. Comparison to the pangenome of species is a tool that can be used to mitigate the risk of overinterpreting results by putting them in a broader context than in a single study. Further work in microbiome strain epidemiology will improve the interpretation of microbiome data.

Other approaches for the detection of antibiotic resistance genes from microbiome samples are available and provide complementary information on the resistome. For example, quantitative PCR allows detection and quantification of the abundance of specific resistance genes, but without determining their sequence or genetic context [33]. Recently, Lanza and collaborators showed that specific sequence capture using a large collection of antibiotic resistance gene probes was a sensitive method to identify 
the resistome of fecal samples and to determine its genomic sequence [43]. One drawback of this approach is that it does not provide the genomic context and taxonomic identification of the origin of these genes. For improved genome context, read-cloud sequencing combined to CIM or CEM methodologies could provide contiguous assemblies of low-abundance genomes [44].

\section{Conclusion}

In this work, we addressed two important issues of microbiome analysis. First, we suggested a strategy to investigate the genome of bacteria of low relative abundance in the gut microbiome without overly deep sequencing. The culture conditions we used for CEM were especially efficient in recovery of Enterobacteriaceae. The use of other liquid culture media could contribute to an improved genotyping of less-abundant gut bacteria, for example, those associated with the mucosa. Second, we interpreted the potential importance of resistance genes from the gut microbiome by exploiting existing genomic information from public databases. As more bacterial genomes become available, it is essential to consider the information they provide in interpreting results from metagenomic studies, not limited to resistance gene analysis. Defining if a gene is part of the core genome of a species is an approach that can help interpret genomes recovered from microbiomes. This big data approach improves the information that can be extracted from microbial population studies.

\section{Additional files}

Additional file 1: Table S1. Sequencing statistics of culture-enriched microbiomes. (XLSX $26 \mathrm{~kb}$ )

Additional file 2: Figure S1. Diversity and abundance distribution of bacterial families in culture-independent or culture-enriched microbiomes. A) Beanplots of the Shannon diversity of CIM or CEM samples profiled at the rank of species. B) Abundance distribution of bacterial families in CIM or CEM. For each curve, the families are ordered from the most abundant to the least abundant (for the 25 first taxa). Data shown are the average proportion of each taxon for each condition. (PDF $432 \mathrm{~kb}$ )

Additional file 3: Table S2. Taxonomic profiling at the rank of species. (XLS $6107 \mathrm{~kb}$ )

Additional file 4: Table S3. Assembled genome size per species. (XLSX $377 \mathrm{~kb}$ )

Additional file 5: Figure S2. Relationship between the depth of sequencing and sum of the assembled contigs associated with a species for 225 species. Each panel represents a different species. The $y$ axis represents the nucleotides assembled into contigs associated with a species. The $x$ axis is the estimated number of nucleotide sequences for the species (proportion of the species in the microbiome multiplied by the number of nucleotides sequenced for this sample). The horizontal lines indicate 1 million nucleotides. As represented in the legend, the color of the points relate to the CIM or CEM conditions from which each point originates. (PDF $3014 \mathrm{~kb}$ )

Additional file 6: Figure S3. Distribution of pathways in cultureindependent or culture-enriched microbiomes. Pathways are considered present in a condition if at least one EC number of this pathway is present in at least one sample of said condition. (PDF $136 \mathrm{~kb}$ )
Additional file 7: Figure S4. Proportion of samples and enzymes positive per cluster per condition. For each combination of enzyme clusters and experimental conditions, the proportion of positive enzymes compared to the total size of the combination was calculated. A value of 1 indicates that all samples were positive for all enzymes. A value of 0 indicates that no sample in a given condition was positive for any enzyme from the cluster. (PDF $305 \mathrm{~kb}$ )

Additional file 8: Table S4. Number of resistance genes observed per culture-independent or culture-enriched microbiomes with 70 percent identity to reference resistance genes from MERGEM. (XLSX 14 kb)

Additional file 9: Table S6. Potential species origin of antibiotic resistance genes. (XLSX $25 \mathrm{~kb}$ )

Additional file 10: Table S7. Families of antibiotic resistance genes associated with potential species of origin. (XLSX $154 \mathrm{~kb}$ )

Additional file 11: Table S5. Number of resistance genes observed per microbiome and selectome with 70 percent identity to reference resistance genes from MERGEM. (XLSX 14 kb)

Additional file 12: Figure S5. Association between genome content clustering and content in resistance genes for 432 Enterobacter cloacae whole genomes. The color of the heatmap indicates the copy number of each resistance gene in whole genomes. Hierarchical clustering of the genomes is based on the comparison of their content in k-mers using the Ray Surveyor software. (PDF 1449 kb)

Additional file 13: Figure S6. Association between genome content clustering and content in resistance genes for 3346 Escherichia coli whole genomes. The color of the heatmap indicates the copy number of each resistance gene in whole genomes. Hierarchical clustering of the genomes is based on the comparison of their content in k-mers using the Ray Surveyor software. (PDF 1465 kb)

Additional file 14: Figure S7. Distance in nucleotides between the core and accessory resistance genes from mobile elements in E. coli and E. cloacae. (PDF $175 \mathrm{~kb}$ )

\section{Funding}

This study was funded by the Québec Consortium for Drug Discovery (CQDM) and by the Canada Research Chair Program (JC and MO). FR is associated to Canada Research Excellence Chair in the MicrobiomeEndocannabinoidome Axis in Metabolic Health. FR, AAO, PLP and MD were supported by Mitacs through the Mitacs-Accelerate program. Computations were performed on the Guillimin supercomputer at McGill University and the Colosse supercomputer at Université Laval, under the auspices of Calcul Québec and Compute Canada.

\section{Availability of data and materials}

Sequence of culture-independent microbiomes (CIM) are available in European Nucleotide Archive PRJEB8094. Sequence of culture-enriched microbiomes (CEM) are available in European Nucleotide Archive PRJEB28237.

\section{Authors' contributions}

FR and MB contributed to the experimental design, sequenced samples, performed bioinformatics and data mining, analyzed the results, and wrote the manuscript. AAO prepared the sequencing libraries. EB managed the stool samples. AAO and EB cultivated the stool samples and extracted the nucleic acids. MD, PLP, SRK, and PHR performed the bioinformatics analyses. $\mathrm{AH}, \mathrm{PHR}, \mathrm{MO}, \mathrm{MHB}$, and $\mathrm{JC}$ offered technical and scientific support for the study. MB, AH, PHR, MO, MGB, and JC designed the study and supervised the project. All authors critically reviewed the manuscript. All authors read and approved the final manuscript.

\section{Ethics approval and consent to participate}

The study protocol was approved by the ethical committee of the $\mathrm{CHU}$ de Québec - Université Laval. Informed written consent was obtained from participants.

\section{Competing interests}

The authors declare that they have no conflicts of interests. 


\section{Publisher's Note}

Springer Nature remains neutral with regard to jurisdictional claims in published maps and institutional affiliations.

\section{Author details fonctionnels, Québec, Canada. ${ }^{3}$ Centre de Recherche en Infectiologie de I'Université Laval, Axe Maladies Infectieuses et Immunitaires, Centre de Recherche du CHU de Québec-Université Laval, Québec City, Canada. Canada. ${ }^{7}$ Département de biochimie, de microbiologie et de City, Canada \\ Received: 11 December 2018 Accepted: 20 March 2019 \\ Published online: 05 April 2019}

'École de nutrition, Faculté des sciences de l'agriculture et de l'alimentation, Université Laval, Québec City, Canada. Institut sur la nutrition et les aliments ${ }^{4}$ Département de Microbiologie, Infectiologie et d'Immunologie, Faculté de Médecine, Université Laval, Québec City, Canada. ${ }^{5}$ Centre de recherche en données massives, Université Laval, Québec City, Canada. ${ }^{6}$ Département de médecine moléculaire, Faculté de Médecine, Université Laval, Québec City, bio-informatique, Faculté des Sciences et de Génie, Université Laval, Québec

\section{References}

1. Bush K, Courvalin P, Dantas G, Davies J, Eisenstein B, Huovinen P, et al. Tackling antibiotic resistance. Nat Rev Microbiol. 2011:9:894-6.

2. Carlet J, Collignon P, Goldmann D, Goossens H, Gyssens IC, Harbarth S, et al. Society's failure to protect a precious resource: antibiotics. Lancet. 2011:378:369-71.

3. Blaser MJ. The Past and Future Biology of the Human Microbiome in an Age of Extinctions. Cell. 2018;172:1173-7.

4. Carlet J. The gut is the epicentre of antibiotic resistance. Antimicrob Resist Infect Control. 2012;1:39.

5. Mack SG, Turner RL, Dwyer DJ. Achieving a Predictive Understanding of Antimicrobial Stress Physiology through Systems Biology. Trends Microbiol. 2018;26:296-312

6. Ferreiro A, Crook N, Gasparrini AJ, Dantas G. Multiscale Evolutionary Dynamics of Host-Associated Microbiomes. Cell. 2018;172:1216-27.

7. Dethlefsen $L$, Relman DA. Incomplete recovery and individualized responses of the human distal gut microbiota to repeated antibiotic perturbation. Proc Natl Acad Sci U S A. 2011;108(Suppl):4554-61.

8. Crofts TS, Gasparrini AJ, Dantas G. Next-generation approaches to understand and combat the antibiotic resistome. Nat Rev Microbiol. 2017; 15:422-34.

9. Raymond F, Ouameur AA, Déraspe M, lqbal N, Gingras H, Dridi B, et al. The initial state of the human gut microbiome determines its reshaping by antibiotics. ISME J. 2016:10:707-20.

10. Rodriguez-R LM, Konstantinidis KT. Estimating coverage in metagenomic data sets and why it matters. ISME J. 2014:1-3.

11. Raymond F, Déraspe M, Boissinot M, Bergeron MG, Corbeil J. Partial recovery of microbiomes after antibiotic treatment. Gut Microbes. 2016:7:428-34.

12. Hiergeist A, Gläsner J, Reischl U, Gessner A. Analyses of Intestinal Microbiota: Culture versus Sequencing. ILAR J. 2015;56:228-40.

13. Hamad I, Ranque S, Azhar El, Yasir M, Jiman-Fatani AA, Tissot-Dupont H, et al. Culturomics and amplicon-based metagenomic approaches for the study of fungal population in human gut microbiota. Sci Rep. 2017;7:16788.

14. Ferrario C, Alessandri G, Mancabelli L, Gering E, Mangifesta M, Milani C, et al. Untangling the cecal microbiota of feral chickens by culturomic and metagenomic analyses. Environ Microbiol. 2017;19:4771-83.

15. Kambouris ME, Pavlidis C, Skoufas E, Arabatzis M, Kantzanou M, Velegraki A, et al. Culturomics: a new kid on the block of OMICS to enable personalized medicine. OMICS. 2018:22:108-18.

16. Lagier J-C, Drancourt M, Charrel R, Bittar F, La Scola B, Ranque S, et al. Many more microbes in humans: enlarging the microbiome repertoire. Clin Infect Dis. 2017;65:S20-9.

17. Lagier JC, Khelaifia S, Alou MT, Ndongo S, Dione N, Hugon P, et al. Culture of previously uncultured members of the human gut microbiota by culturomics. Nat Microbiol. 2016;1:16203.

18. Lagier J-C, Armougom F, Million M, Hugon P, Pagnier I, Robert C, et al. Microbial culturomics: paradigm shift in the human gut microbiome study. Clin Microbiol Infect. 2012;18:1185-93.

19. Lau JT, Whelan FJ, Herath I, Lee CH, Collins SM, Bercik P, et al. Capturing the diversity of the human gut microbiota through culture-enriched molecular profiling. Genome Med. 2016;8:72.
20. Rettedal EA, Gumpert H, Sommer MOA. Cultivation-based multiplex phenotyping of human gut microbiota allows targeted recovery of previously uncultured bacteria. Nat Commun. 2014;5:4714.

21. Auchtung JM, Robinson CD, Britton RA. Cultivation of stable, reproducible microbial communities from different fecal donors using minibioreactor arrays (MBRAs). Microbiome. 2015;3:42.

22. Domingo M-C, Huletsky A, Giroux R, Picard FJ, Bergeron MG. vanD and vanG-like gene clusters in a Ruminococcus species isolated from human bowel flora. Antimicrob Agents Chemother. 2007;51:4111-7.

23. Goodman AL, Kallstrom G, Faith JJ, Reyes A, Moore A, Dantas G, et al. Extensive personal human gut microbiota culture collections characterized and manipulated in gnotobiotic mice. Proc Natl Acad Sci U S A. 2011;108:6252-7.

24. Boisvert S, Laviolette F, Corbeil J. Ray: Simultaneous assembly of reads from a mix of high-throughput sequencing technologies. J Comput Biol. 2010;17: 1519-33.

25. Boisvert S, Raymond F, Godzaridis E, Laviolette F, Corbeil J. Ray Meta: scalable de novo metagenome assembly and profiling. Genome Biol. BioMed Central Ltd. 2012;13:R122

26. Fu L, Niu B, Zhu Z, Wu S, Li W. CD-HIT: accelerated for clustering the nextgeneration sequencing data. Bioinformatics. 2012;28:3150-2.

27. Chang A, Schomburg I, Placzek S, Jeske L, Ulbrich M, Xiao M, et al. BRENDA in 2015: exciting developments in its 25th year of existence. Nucleic Acids Res. 2015:43:D439-46.

28. Albenberg L, Esipova TV, Judge CP, Bittinger K, Chen J, Laughlin A, et al. Correlation Between Intraluminal Oxygen Gradient and Radial Partitioning of Intestinal Microbiota. Gastroenterology. 2014;147:1055-1063.e8.

29. Lloyd-Price J, Mahurkar A, Rahnavard G, Crabtree J, Orvis J, Hall AB, et al. Strains, functions and dynamics in the expanded Human Microbiome Project. Nature. 2017;550:61-6.

30. Pehrsson EC, Tsukayama P, Patel S, Mejía-Bautista M, Sosa-Soto G, Navarrete $\mathrm{KM}$, et al. Interconnected microbiomes and resistomes in low-income human habitats. Nature. 2016;533:212-6.

31. Polz MF, Alm EJ, Hanage WP. Horizontal gene transfer and the evolution of bacterial and archaeal population structure. Trends Genet. 2013;29:170-5.

32. Moradigaravand D, Boinett CJ, Martin V, Peacock SJ, Parkhill J. Recent independent emergence of multiple multidrug-resistant Serratia marcescens clones within the United Kingdom and Ireland. Genome Res. 2016:26:1101-9.

33. Do TT, Tamames J, Stedtfeld RD, Guo X, Murphy S, Tiedje JM, et al. Antibiotic resistance gene detection in the microbiome context. Microb Drug Resist. 2018;24:542-6.

34. Claros MC, Citron DM, Goldstein EJ. Survival of anaerobic bacteria in various thioglycolate and chopped meat broth formulations. J Clin Microbiol. 1995; 33:2505-7.

35. Lewis JP, lyer D, Anaya-Bergman C. Adaptation of porphyromonas gingivalis to microaerophilic conditions involves increased consumption of formate and reduced utilization of lactate. Microbiology. 2009;155:3758-74.

36. Donaldson GP, Lee SM, Mazmanian SK. Gut biogeography of the bacterial microbiota. Nat Rev Microbiol. 2016;14:20-32.

37. Maurice CF, Haiser HJ, Turnbaugh PJ. Xenobiotics shape the physiology and gene expression of the active human gut microbiome. Cell. 2013:152:39-50.

38. Brito IL, Yilmaz S, Huang K, Xu L, Jupiter SD, Jenkins AP, et al. Mobile genes in the human microbiome are structured from global to individual scales. Nature. 2016:535:435-9.

39. Forsberg KJ, Patel S, Gibson MK, Lauber CL, Knight R, Fierer N, et al. Bacterial phylogeny structures soil resistomes across habitats. Nature. 2014;509:612-6.

40. Fernández $\mathrm{L}$, Hancock REW. Adaptive and mutational resistance: role of porins and efflux pumps in drug resistance. Clin Microbiol Rev. 2012;25:661-81.

41. Martínez JL, Coque TM, Baquero F. What is a resistance gene? Ranking risk in resistomes. Nat Rev Microbiol. 2015:13:116-23.

42. Jacoby GA. AmpC beta-lactamases. Clin Microbiol Rev. 2009;22:161-82.

43. Lanza VF, Baquero F, Martínez JL, Ramos-Ruíz R, González-Zorn B, Andremont $A$, et al. In-depth resistome analysis by targeted metagenomics. Microbiome. 2018;6:11.

44. Bishara A, Moss EL, Kolmogorov M, Parada AE, Weng Z, Sidow A, et al. Highquality genome sequences of uncultured microbes by assembly of read clouds. Nat Biotechnol. 2018:36:1067-75. 